\title{
Correction to: Development of a survivorship screening tool in survivors of hematopoietic cell transplant
}

\author{
Jennifer A. Hydeman ${ }^{1}$ - Megan E. Pailler ${ }^{1}$ - Pamela D. Paplham ${ }^{2} \cdot$ Michael A. Zevon ${ }^{1}$
}

Published online: 16 July 2021

(C) Springer Science+Business Media, LLC, part of Springer Nature 2021

\section{Correction to: Journal of Cancer Survivorship https://doi.org/10.1007/s11764-021-01043-5}

The original version of the article has contained a mistake in table 1,2 and result section.

The following changes: It replaces the bolding in Table 1 with new bolding to indicate the highest factor loadings. The note following Table 1 should read as follows, "Note: Highest factor loadings for each item are in bold. All items were retained with a factor loading of $\geq 0.4$ ".

The title for Table 2 should read: "Screening tool questions with clinical cutoffs indicating need for referral". The text following this should sit above the table as directions included in the questionnaire.

The following key should be included under Table 2:

The online version of the original article can be found at https://doi.org/ 10.1007/s11764-021-01043-5

Jennifer A. Hydeman

jennifer.hydeman@ roswellpark.org

1 Department of Psychology, Roswell Park Comprehensive Cancer

Center, Buffalo, NY 14263, USA

2 Department of Nursing Administration, Roswell Park

Comprehensive Cancer Center, Buffalo, NY 14263, USA
Key:

Items 1-7, 12-14 Psychosocial oncology.

Item 8 AYA

Items 9 -10 Case management

Items 11 Pastoral Care

Items 14-17 Physical Therapy

Items 18-22 Occupational Therapy

Items 23-25. Nutrition

The final correction is in the last line of the Results section. It currently reads "red" it should read "bold".

The updated table 1 and 2 is give below. 
Table 1 Exploratory factor analysis $(\mathrm{n}=254)$

\begin{tabular}{|c|c|c|c|}
\hline Item & $\begin{array}{l}\text { Factor } 1 \\
\text { Physical/functional }(\alpha=0.89)\end{array}$ & $\begin{array}{l}\text { Factor } 2 \\
\text { Emotional }(\alpha=0.71)\end{array}$ & $\begin{array}{l}\text { Factor } 3 \\
\text { Practical }(\alpha=0.72)\end{array}$ \\
\hline 1. Transportation & 0.08 & -0.20 & 0.74 \\
\hline 2. Finances & -0.21 & 0.70 & 0.30 \\
\hline 3. Managing daily concerns & 0.18 & 0.37 & 0.46 \\
\hline 4. Worry about the future & 0.46 & 0.78 & -0.04 \\
\hline 5. Community resources & 0.18 & 0.02 & 0.70 \\
\hline 6. Substance use & -0.03 & 0.45 & -0.14 \\
\hline 7. Feeling isolated & 0.08 & 0.33 & 0.46 \\
\hline 8. Emotional distress & 0.32 & 0.51 & -0.04 \\
\hline 9. Sexuality/intimacy & 0.18 & 0.50 & -0.20 \\
\hline 10. Fertility & -0.08 & 0.04 & 0.49 \\
\hline 11. Obtaining medicines & -0.07 & 0.55 & 0.14 \\
\hline 12. Insurance & -0.15 & 0.50 & 0.38 \\
\hline 13. Spirituality & -0.22 & 0.08 & 0.67 \\
\hline 14. Weakness & 0.86 & 0.01 & -0.05 \\
\hline 15. Poor movement & 0.76 & 0.01 & 0.12 \\
\hline 16. Maintaining balance & 0.67 & -.001 & 0.03 \\
\hline 17. Trouble walking & 0.71 & 0.02 & 0.08 \\
\hline 18. Caring for self & 0.60 & -0.32 & 0.42 \\
\hline 19. Trouble using arms & 0.51 & 0.13 & 0.18 \\
\hline 20. Memory concerns & 0.53 & 0.33 & -0.10 \\
\hline 21. Fatigue & 0.69 & 0.35 & -0.13 \\
\hline 22. Fatigue interfering & 0.67 & 0.36 & -0.04 \\
\hline 23. Losing weight & 0.55 & -0.11 & -0.10 \\
\hline 24. Loss of appetite & 0.43 & -0.16 & 0.40 \\
\hline 25. Nausea & 0.58 & -0.14 & -0.11 \\
\hline
\end{tabular}

Note: Highest factor loadings for each item are in bold. All items were retained with a factor loading of $\geq 0.4$ 
Table 2 Screening tool questions

Please circle the number to indicate how much each of the following issues have been a problem for you. An answer of " 0 " means that this is not a problem at all for you. An answer of "4" means that it is very much a problem for you

\begin{tabular}{|c|c|c|c|c|c|c|}
\hline 1 & Transportation or local lodging during treatment & 0 & 1 & 2 & 3 & 4 \\
\hline & & Not a problem & A little bit & Somewhat & Quite a bit & Large problem \\
\hline \multirow[t]{2}{*}{2} & Finances & 0 & & & & 4 \\
\hline & & Not a problem & A little bit & Somewhat & Quite a bit & Large problem \\
\hline \multirow[t]{2}{*}{3} & Managing work, school, or home life & 0 & & & & 4 \\
\hline & & Not a problem & A little bit & Somewhat & Quite a bit & Large problem \\
\hline \multirow[t]{2}{*}{4} & Worry about the future & 0 & & & & 4 \\
\hline & & Not a problem & A little bit & Somewhat & Quite a bit & Large problem \\
\hline \multirow[t]{2}{*}{5} & Finding community resources near where I live & 0 & & & & 4 \\
\hline & & Not a problem & A little bit & Somewhat & Quite a bit & Large problem \\
\hline \multirow[t]{2}{*}{6} & Substance use- you or in your environment & 0 & & & & 4 \\
\hline & (drugs, alcohol, nicotine, prescriptions, etc.) & Not a problem & A little bit & Somewhat & Quite a bit & Large problem \\
\hline \multirow[t]{2}{*}{7} & Feeling isolated, alone or abandoned & 0 & & 2 & 3 & 4 \\
\hline & & Not a problem & A little bit & Somewhat & Quite a bit & Large problem \\
\hline \multirow[t]{2}{*}{8} & Concerns about fertility & 0 & & & & 4 \\
\hline & & Not a problem & A little bit & Somewhat & Quite a bit & Large problem \\
\hline \multirow[t]{2}{*}{9} & Getting medicines & 0 & & & & 4 \\
\hline & & Not a problem & A little bit & Somewhat & Quite a bit & Large problem \\
\hline \multirow[t]{2}{*}{10} & Health insurance & 0 & & & & 4 \\
\hline & & Not a problem & A little bit & Somewhat & Quite a bit & Large problem \\
\hline \multirow[t]{2}{*}{11} & Spiritual or religious concerns & 0 & & & & 4 \\
\hline & & Not a problem & A little bit & Somewhat & Quite a bit & Large problem \\
\hline 12 & $\begin{array}{l}\text { How much emotional distress have you been } \\
\text { experiencing in the past week including today? }\end{array}$ & $\begin{array}{l}0 \\
\text { No distress }\end{array}$ & 123 & 456 & 789 & 10 \\
\hline 13 & $\begin{array}{l}\text { Please circle the number }(0-10) \text { that best describes the } \\
\text { extent to which your cancer or cancer treatment } \\
\text { have caused problems related to sexuality or intimacy }\end{array}$ & $\begin{array}{l}0 \\
\text { No problem }\end{array}$ & 123 & 456 & 789 & $\begin{array}{l}10 \\
\text { Severe problem }\end{array}$ \\
\hline \multirow[t]{2}{*}{14} & Weakness in my arms or legs & 0 & 1 & 2 & 3 & 4 \\
\hline & & Not a problem & A little bit & Somewhat & Quite a bit & Large problem \\
\hline \multirow[t]{2}{*}{15} & Poor movement in my legs/feet & 0 & & & & 4 \\
\hline & & Not a problem & A little bit & Somewhat & Quite a bit & Large problem \\
\hline \multirow[t]{2}{*}{16} & Maintaining my balance/falls & 0 & & & & 4 \\
\hline & & Not a problem & A little bit & Somewhat & Quite a bit & Large problem \\
\hline \multirow[t]{2}{*}{17} & Trouble walking & & & & & 4 \\
\hline & & Not a problem & A little bit & Somewhat & Quite a bit & Large problem \\
\hline 18 & $\begin{array}{l}\text { Trouble caring for myself (bathing, } \\
\text { dressing, eating, meal preparation } \\
\text { or light housekeeping) }\end{array}$ & $\begin{array}{l}0 \\
\text { Not a problem }\end{array}$ & A little bit & $\begin{array}{l}2 \\
\text { Somewhat }\end{array}$ & $\begin{array}{l}3 \\
\text { Quite a bit }\end{array}$ & $\begin{array}{l}4 \\
\text { Large problem }\end{array}$ \\
\hline \multirow[t]{2}{*}{19} & Trouble using my arms and hands to & 0 & & 2 & 3 & 4 \\
\hline & $\begin{array}{l}\text { complete everyday activities } \\
\text { (e.g. joint limitations, weakness, } \\
\text { tremors, numbness) }\end{array}$ & Not a problem & A little bit & Somewhat & Quite a bit & Large problem \\
\hline \multirow[t]{2}{*}{20} & Trouble with my memory and/or concentration & 0 & 1 & 2 & 3 & 4 \\
\hline & & Not a problem & A little bit & Somewhat & Quite a bit & Large problem \\
\hline 21 & $\begin{array}{l}\text { Please rate your fatigue (weariness, tiredness) by } \\
\text { circling the number that best describes your } \\
\text { fatigue over the past } 7 \text { days }\end{array}$ & $\begin{array}{l}0 \\
\text { No fatigue }\end{array}$ & 123 & 456 & 789 & $\begin{array}{l}10 \\
\text { Most fatigue }\end{array}$ \\
\hline \multirow[t]{2}{*}{22} & Please circle the number $(0-10)$ that describes how fatigue & 0 & 123 & 456 & 789 & 10 \\
\hline & $\begin{array}{l}\text { has interfered with your normal work (includes both work } \\
\text { outside the home and daily chores) in the past } 7 \text { days }\end{array}$ & $\begin{array}{l}\text { Does not get in } \\
\text { the way }\end{array}$ & & & & Totally gets in the way \\
\hline \multirow[t]{2}{*}{23} & Losing weight without trying & 0 & 1 & 2 & 3 & 4 \\
\hline & & Not a problem & A little bit & Somewhat & Quite a bit & Large problem \\
\hline 24 & Loss of appetite & 0 & & & & 4 \\
\hline & & Not a problem & A little bit & Somewhat & Quite a bit & Large problem \\
\hline 25 & Experiencing nausea, vomiting, diarrhea, or constipation & & & & & 4 \\
\hline & & Not a problem & A little bit & Somewhat & Quite a bit & Large problem \\
\hline
\end{tabular}

Key:

Items 1-7, 12-14 Psychosocial oncology.

Item 8 AYA

IItems 9 -10 Case management

Items 11 Pastoral Care

Items 14-17 Physical Therapy

Items18-22 Occupational Therapy

Items 23-25. Nutrition

Publisher's note Springer Nature remains neutral with regard to jurisdictional claims in published maps and institutional affiliations. 